\title{
Synthesis of 2,4,6,8,10-pentaaza[3.3.3]propellane substituted with different groups
}

\author{
Lizhu Zhang ${ }^{1}$, Qi Zhang ${ }^{1}$, Yahan Xue ${ }^{1}$, Guangwei Zhou ${ }^{1}$, Qunsheng Yan ${ }^{1}$ and Jiarong Li $^{1 *}$ \\ ${ }^{1}$ Chemistry and Chemical Engineering, Beijing Institute of Technology, Beijing 100081, China
}

\begin{abstract}
Polyaza[3.3.3]propellanes have good symmetry and strong ring tension, which are suitable as the skeleton structure of functional materials such as high energy density materials. Based on the single benzyl derivative of 3,7,9.11-tetraoxo-2,4,6,8,10-pentaaza[3.3.3]propellane (compound 3 ), a series of 2,4,6,8,10pentaaza[3.3.3]propellane derivatives, such as $\mathbf{4 a - c}$ and their reduced derivatives 5a-c, were synthesized successfully.
\end{abstract}

\section{Introduction}

Tricyclic propellanes are a kind of polycyclic compounds in which three ring systems share a single bond (Figure 1) [1-5]. They have high reactivity and exist in many natural products [6-13]. Since the propellanes were firstly reported by Ginsburg [14] in 1965, they have aroused the interest of many chemists [15-17]. Nitrocyclopropellanes, a class of heterocyclic propellanes, can be easily transformed to N-substituted compounds due to the high reactivity of nitrogen atoms, and they have attracted the attention of many researchers [18-20].

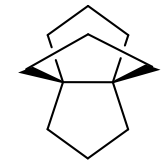

[3.3.3] propellane

(a)

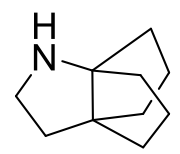

2-aza[3.3.3] propellane

(b)<smiles>C1CCC23COCCC2(C1)C3</smiles>

3-oxa[4.4.3]propellane

(c)

Figure. 1 The structure of propellanes.

Because aza[3.3.3]propellanes have good symmetry and compact skeleton, they have good compactness. In 1966, Altman [2] et al first synthesized monoaza[3.3.3]propellane and diaza[3.3.3] propellane, which enabled researchers to started the design and synthesis of azapropellanes. Combined with the influence of space tension on enthalpy of formation, detonation rate and work ability, they are ideal structures of energycontain-ing compounds. The secondary amine in the azapropellanes structure provides an active reaction site for the further introduction of energetic groups, and further reactions to obtain nitrogensubstituted compounds [21].

Recently, Burnett [18] reported the synthesis of 3,7,9,11-tetraoxo-2,4,6,8,10-pentaaza[3.3.3] propellane with monosubsti-tuted alkyl groups. Kim [22] synthesized 2,4,6,8,10-pentaaza[3.3.3] propellanes substituted with same groups (Scheme 1) by the condensation of 3,7,9.11-tetraoxo-2,4,6,8,10pentaaza[3.3.3]propellane with benzylamine in 2014 . However, 2,4,6,8,10-pentaaza[3.3.3] propellane derivatives substituted with different groups have never been reported. In order to study the debenzylation of pentaaza[3.3.3]propellane, we need derivatives substituted with different groups at nitrogen atoms. Therefore, we describe the preparation of N-substituted pentaazapropellanes with different substituents (Scheme 2). 


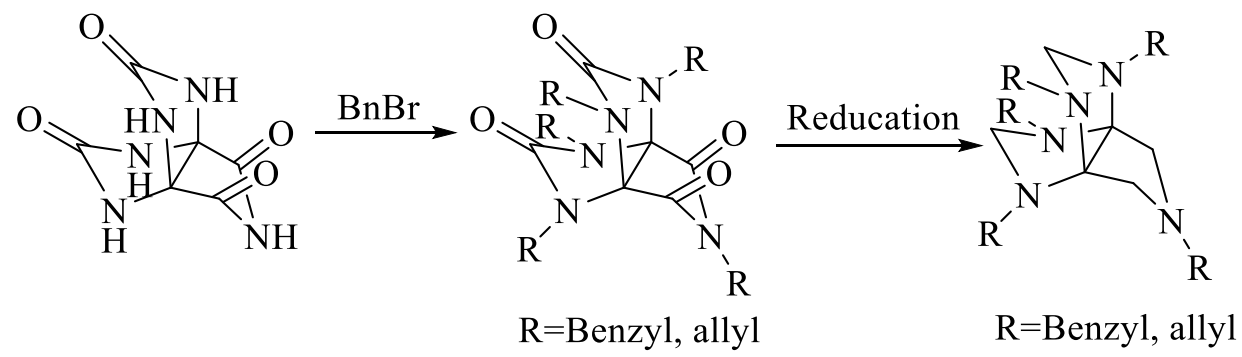

Scheme. 1 Synthesis of 2,4,6,8,10-pentaaza[3.3.3]propellane substituted with same groups.

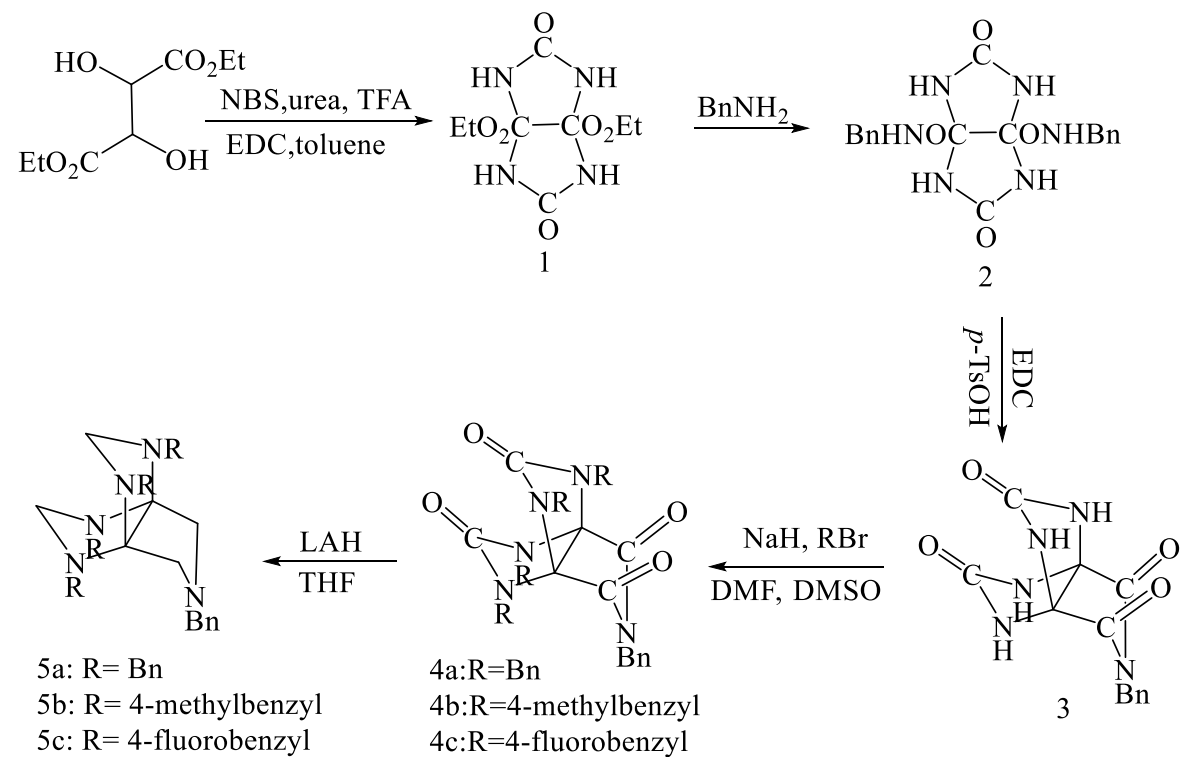

Scheme 2. Synthesis of 2,4,6,8,10-pentaaza[3.3.3]propellane substituted with different groups.

\section{Results and discussion}

Compound 1 was prepared by bromination and urea condensation with diethyl tartrate as raw material according to the literature 22 , but it was worth mentioning that the yield of compound 1 increased by $30 \%$ here. Compound $\mathbf{1}$ is difficult to dissolve in most organic solvents, however, when the amount of benzylamine used as a solvent and reactant was increased, compound $\mathbf{1}$ was successfully transformed to compound $\mathbf{2}$ in high yield.

Followed by benzylation, 10-benzyl derivative of 3,7,9.11-tetraoxo-2,4,6,8,10-pentaaza[3.3.3] propellane 3 was easy to be obtained by refluxing of dichloromethane solution of benzylation mixture in the present of $p$ toluenesulfonic acid.

With single benzyl derivatives in hand, 3,7,9,11tetraoxo-2,4,6,8,10-pentabenzyl-2,4,6,8,10-pentaaza [3.3.3]propellane $\mathbf{4 a}$ was obtained by the reaction of $\mathbf{3}$ with $\mathrm{BnBr}$ in the present of $\mathrm{NaH}$. Similarly, the other substituted derivatives of 3,7,9,11-tetraoxo-2,4,6,8,10pentaaza[3.3.3]propellane $(\mathbf{4 b}, \mathbf{4 c})$ were provided by replacing benzyl bromide with 4-methylbromo-benzyl and 4-fluorobromobenzyl. During the synthesis of compound $\mathbf{4 a}$, it was found that the solvent plays a crucial role. And systematic research showed that when the amination was operated in a mixed solvent (DMSO: DMF $=1: 4$ ), the yield of total substituted products of nitrogen of $\mathbf{4}$ was the highest as shown in Table 1.

Table 1. Effect of solvent ratio of DMSO to DMF on the yield of compound 4a.

\begin{tabular}{ccc}
\hline Enty & $\mathrm{V}_{\text {DMSO }}: \mathrm{V}_{\text {DMF }}$ & Yield \\
\hline $\mathbf{1}$ & $1: 0$ & $41 \%$ \\
$\mathbf{2}$ & $1: 1$ & $56 \%$ \\
$\mathbf{3}$ & $1: 2$ & $64 \%$ \\
$\mathbf{4}$ & $1: 3$ & $75 \%$ \\
$\mathbf{5}$ & $1: 4$ & $81 \%$ \\
$\mathbf{6}$ & $1: 5$ & $77 \%$ \\
$\mathbf{7}$ & $1: 6$ & $63 \%$ \\
$\mathbf{8}$ & $0: 1$ & $71 \%$ \\
\hline
\end{tabular}


In order to reduce the carbonyl group of compounds 4, we tried different reducing agents such as $10 \% \mathrm{Pd} / \mathrm{C}$, $\mathrm{LiAlH}_{4}, \mathrm{NaBH}_{4}$ and red aluminum. As a result, it was found that 5a-c were obtained by reduction of compounds 4a-c with lithium aluminum hydride (LAH) in THF.

\section{Experimental}

\subsection{Synthesis of glycoluric diethyl ester (1)}

Diethyl tartrate (50 mmol, $10.30 \mathrm{~g}$ ) and NBS (125 mmol, $22.25 \mathrm{~g}$ ) were added to a $250 \mathrm{~mL}$ round bottom flask, then $80 \mathrm{~mL} \mathrm{1,2-dichloroethane} \mathrm{was} \mathrm{added} \mathrm{as} \mathrm{a} \mathrm{solvent,}$ stirred and refluxed under argon. After 4 hours, the solution was cooled to room temperature, adding anhydrous sodium sulfite to the solution. The mixture was distilled to remove the solvent under reduced pressure, adding $40 \mathrm{~mL}$ ethyl acetate to dilute the mixture. The solid was filtered out, and brown organic phase was removed in vacuum distillation to give a white oily liquid. A solution of this oily liquid, trifluoroacetic acid (218 mmol, $16.5 \mathrm{~mL})$ and urea (100 mmol, $6.00 \mathrm{~g})$ in $80 \mathrm{~mL}$ toluene, were refluxed under argon atmosphere. After 6 hours, the solvent was removed in vacuum distillation and the concentrated solution was stirred for 3 hours in $100 \mathrm{~mL}$ ethanol. The mixture was filtered to give a white powder solid 1 (85\%).

m.p. $294-296{ }^{\circ} \mathrm{C},{ }^{1} \mathrm{H}$ NMR (400 MHz, DMSO-d $d_{6}$, $\left.25^{\circ} \mathrm{C}\right) \delta 8.03(\mathrm{~s}, 4 \mathrm{H}), 4.10(\mathrm{q}, 4 \mathrm{H}, J=8.0 \mathrm{~Hz}), 1.18(\mathrm{t}$, $6 \mathrm{H}, J=8.0 \mathrm{~Hz}) ;{ }^{13} \mathrm{C}$ NMR $\left(100 \mathrm{MHz}, \mathrm{DMSO}-d_{6}, 25^{\circ} \mathrm{C}\right)$ $\delta 167.47$ (s), 159.67, 78.00, 62.13, 13.92; IR (KBr, v/cm1) $3231,1754,1698,1488,1391,1268,1173,1078,1031$, 860, 767; HRMS (ESI ${ }^{+}$: calcd. for $\mathrm{C}_{10} \mathrm{H}_{15} \mathrm{~N}_{4} \mathrm{O}_{6}[\mathrm{M}+\mathrm{H}]^{+}$ 287.0992, found 287.1001 .

\subsection{N, N'-Dibenzyl glycolide diamide (2)}

Glycoluric diethyl ester 1 (20 mmol, $5.72 \mathrm{~g}$ ) was added in a round bottom flask containing $60 \mathrm{~mL}$ benzylamine, and the mixture was refluxed under argon atmosphere. When the reaction was completed, $100 \mathrm{~mL}$ ethanol was added to the resulting mixture. The mixture was stirred at room temperature for 30 minutes to obtain compound 2 . $(98 \%)$.

m. p. $>300{ }^{\circ} \mathrm{C} ;{ }^{1} \mathrm{H}$ NMR $\left(400 \mathrm{MHz}, \mathrm{DMSO}-d_{6}, 25^{\circ} \mathrm{C}\right)$ $\delta 8.22(\mathrm{~s}, 2 \mathrm{H}), 7.64(\mathrm{~s}, 4 \mathrm{H}), 7.27(\mathrm{t}, J=11.7 \mathrm{~Hz}, 10 \mathrm{H})$, $4.13(\mathrm{~d}, J=5.0 \mathrm{~Hz}, 4 \mathrm{H}) ;{ }^{13} \mathrm{C}$ NMR $(100 \mathrm{MHz}$, DMSO$\left.d_{6}, 25{ }^{\circ} \mathrm{C}\right) \delta 167.36,161.16,139.17,128.54,128.07$, 127.22, 79.62, 43.44; IR $\left(\mathrm{KBr}, v / \mathrm{cm}^{-1}\right) 3343,3297$, 2928, 1719, 1697, 1529, 1454, 1360, 1161; HRMS $\left(\mathrm{ESI}^{+}\right.$): calcd. for $\mathrm{C}_{20} \mathrm{H}_{21} \mathrm{~N}_{6} \mathrm{O}_{4}[\mathrm{M}+\mathrm{H}]^{+} 409.1624$, found 409.1626 .

\section{$3.3 \quad 3,7,9.11-T e t r a o x o-10$-benzyl-2,4,6,8,10- pentaaza[3.3.3] propellane (3)}

A mixture of compound 2 (10 mmol, $4.08 \mathrm{~g}$ ), $p$-TsOH (30 mmol, $5.17 \mathrm{~g}$ ) and $60 \mathrm{~mL} \mathrm{1,2-dichloroethane} \mathrm{was}$ refluxed under argon atmosphere. When the reaction was completed, the solvent was removed under reduced pressure. The residue was stirred in $100 \mathrm{~mL}$ ethanol for 3 hours. The white solid was collected by filtration and washed three times with ethanol. The solid was dried under vacuum to obtain compound 3 (97\%).

m. p. $>300{ }^{\circ} \mathrm{C} ;{ }^{1} \mathrm{H}$ NMR $\left(400 \mathrm{MHz}, \mathrm{DMSO}-d_{6}\right.$, $\left.25{ }^{\circ} \mathrm{C}\right) \delta 8.76(\mathrm{~s}, 4 \mathrm{H}), 7.44-7.14(\mathrm{~m}, 5 \mathrm{H}), 4.65(\mathrm{~s}, 2 \mathrm{H})$; ${ }^{13} \mathrm{C}$ NMR $\left(100 \mathrm{MHz}\right.$, DMSO- $\left.d_{6}, 25^{\circ} \mathrm{C}\right) \delta 170.82,159.37$, 135.72, 129.27, 128.38, 127.82, 74.33, 42.07; IR (KBr , $\left.v / \mathrm{cm}^{-1}\right) 3246,2920,1723,1682$; HRMS $\left(\mathrm{ESI}^{+}\right)$: calcd for $\mathrm{C}_{13} \mathrm{H}_{11} \mathrm{~N}_{5} \mathrm{O}_{4}[\mathrm{M}+\mathrm{H}]^{+}$302.0884, found 302.0871 .

\subsection{3,7,9,11-Tetraoxo-2,4,6,8,10-pentabenzyl- 2,4,6,8,10-penta-aza[3.3.3]propellane (4a)}

Compound 3 (10 mmol, $3.01 \mathrm{~g})$ and $\mathrm{BnBr}(60 \mathrm{mmol}$, $10.26 \mathrm{~g}$ ) were dissolved in a mixture of solvents ( $\mathrm{V}_{\text {DMSO }}$ : $\left.\mathrm{V}_{\mathrm{DMF}}=1: 4\right)$ under argon atmosphere. The reaction temperature was cooled using an ice bath. After 5 minutes, $\mathrm{NaH}$ (50 mmol, $2.00 \mathrm{~g})$ was added and the resulting mixture was stirred for $1 \mathrm{~h}$. Then the reaction temperature was raised to room temperature and the reaction was continued for another $12 \mathrm{~h}$. When the end of the reaction was detected, reaction mixture was cooled again and diluted with diethyl ether $(30 \mathrm{~mL})$. A solution of aq $\mathrm{HCl}$ was slowly added to the mixture to quench the unreacted hydride. The resulting mixture was added $\mathrm{H}_{2} \mathrm{O}$ and extracted with ethyl acetate. The combined organic layers were dried with $\mathrm{MgSO}_{4}$, filtered, and concentrated under reduced pressure. The residue oil was purified by silica gel column chromatography, to provide the compound $4 \mathbf{a}$ as a white solid (81\%).

m. p. $151-152{ }^{\circ} \mathrm{C} ;{ }^{1} \mathrm{H}$ NMR $\left(400 \mathrm{MHz}, \mathrm{CDCl}_{3}, 25^{\circ} \mathrm{C}\right)$ $\delta$ 7.84-6.43 (m, 25H), $4.44(\mathrm{~s}, 8 \mathrm{H}), 4.26(\mathrm{~s}, 2 \mathrm{H}) ;{ }^{13} \mathrm{C}$ $\mathrm{NMR}\left(100 \mathrm{MHz}, \mathrm{CDCl}_{3}, 25^{\circ} \mathrm{C}\right) \delta 166.01(\mathrm{~s}), 156.05$ (s), 135.71 (s), 132.56 (s), 127.90 (s), 127.48 (d, $J=33.3 \mathrm{~Hz})$, 126.56 (s), 125.99 (s), 74.39 (s), 44.65 (s), 41.62 (s); IR $\left(\mathrm{KBr}, \mathrm{v} / \mathrm{cm}^{-1}\right)$ 2925, 1718, 1690, 1446, 1146, 936, 694; HRMS $\left(\mathrm{ESI}^{+}\right)$: calcd. for $\mathrm{C}_{41} \mathrm{H}_{36} \mathrm{~N}_{5} \mathrm{O}_{4}[\mathrm{M}+\mathrm{H}]^{+} 662.2762$, found 662.2779 .

\subsection{3,7,9,11-Tetraoxo-2,4,6,8-(4-methyl)benzyl- 10-benzyl-2,4,6, 8,10-pentaaza[3.3.3] propellane (4b)}

4-Me-bnBr was replace of $\mathrm{BnBr}$, the synthesis of compound $\mathbf{4 b}(76 \%)$ was the same as the compound $4 \mathbf{a}$

m.p. $172.4-172.9{ }^{\circ} \mathrm{C}$; ${ }^{1} \mathrm{H}$ NMR $\left(400 \mathrm{MHz}, \mathrm{CDCl}_{3}\right.$, $\left.25{ }^{\circ} \mathrm{C}\right) \delta 7.37-6.69(\mathrm{~m}, 21 \mathrm{H}), 4.41(\mathrm{~s}, 8 \mathrm{H}), 4.25(\mathrm{~d}, J=$ $14.7 \mathrm{~Hz}, 2 \mathrm{H}), 2.22$ (s, 12H); ${ }^{13} \mathrm{C} \mathrm{NMR}\left(100 \mathrm{MHz}, \mathrm{CDCl}_{3}\right.$ $\left.25{ }^{\circ} \mathrm{C}\right) \delta 166.13(\mathrm{~s}), 156.07$ (s), 136.04 (s), 132.69 (d), 128.26 (s), 127.65 (d), 127.17 (s), 125.97 (s), 74.32(s), 44.38(s), 41.52(s), 20.09(d); IR (KBr, v/ $\left.\mathrm{cm}^{-1}\right) 3086.11$, 2962.66, 2924.09, 1716.65, 1456.26, 1386.82, 1336.67, 1244.09, 1168.86, 1139.93, 1109.07, 991.41, 948.98, 916.19, 866.04, 729.09, 524.64; HRMS (ESI $\left.{ }^{+}\right)$: calcd. for $\mathrm{C}_{45} \mathrm{H}_{43} \mathrm{~N}_{5} \mathrm{O}_{4}[\mathrm{M}+\mathrm{H}]^{+} 718.3388$, found 718.3378 . 


\section{$3.6 \quad 3,7,9,11-T e t r a o x o-2,4,6,8-(4-$ fluoro)benzyl- 10-benzyl-2,4,6, 8,10- pentaaza[3.3.3]propellane $(4 \mathrm{c})$}

4-F-bnBr was replace of $\mathrm{BnBr}$, the synthesis of compound $4 \mathrm{c}(68 \%)$ was the same as the compound $\mathbf{4 a}$

m.p. $108.4-110.1^{\circ} \mathrm{C} ;{ }^{1} \mathrm{H}$ NMR (400 MHz, DMSO, $\left.25^{\circ} \mathrm{C}\right) \delta 7.55-6.49(\mathrm{~m}, 21 \mathrm{H}), 5.06-4.11(\mathrm{~m}, 10 \mathrm{H}) .{ }^{13} \mathrm{C}$ NMR (100 MHz, DMSO, $\left.25^{\circ} \mathrm{C}\right) \delta 167.19$ (s), 162.99 (s), 160.57 (s), 157.26 (s), 134.79 (s), 133.58 (d), 129.53 $128.96(\mathrm{~m}), 128.33$ (s), 115.58 (d), 115.41 - 115.23 (m), 75.16 (s), 44.82 (s); IR (KBr, v/ $\left.\mathrm{cm}^{-1}\right)$ 3068.75, 2924.09, $1720.50,1606.70,1510.26,1456.26,1411.89,1342.46$, $1232.51,1147.65,1099.43,947.05,844.82,815.89$, 736.81, 698.23, 665.44, 526.57, 491.85; HRMS (ESI ${ }^{+}$): calcd. for $\mathrm{C}_{41} \mathrm{H}_{31} \mathrm{~F}_{4} \mathrm{~N}_{5} \mathrm{O}_{4}[\mathrm{M}+\mathrm{H}]^{+} 734.2385$, found 734.2374 .

\section{$3.7 \quad 2,4,6,8,10$-Pentabenzyl-2,4,6,8,10- pentaaza[3.3.3]propellane (5a)}

Compound 4a was dissolved in $50 \mathrm{~mL}$ THF, cooling the solution to $0{ }^{\circ} \mathrm{C}$ with an ice bath. After 5 minutes, LAH (60 mmol, $2.28 \mathrm{~g}$ ) was added slowly at low temperatures, under refluxing for $12 \mathrm{~h}$. When the reaction was completed, the reaction mixture was cooled to $0{ }^{\circ} \mathrm{C}$ again. Ethyl acetate $(6 \mathrm{~mL})$ was slowly added to the reaction mixture and the resulting mixture was stirred for 30 minutes to quench the residual hydride. A solution of aq $\mathrm{NaOH}(10 \%, 12 \mathrm{~mL})$ was added until the sludgy phase disappeared. After stirring for another 20 minutes, the mixture was filtered with EtOAc and the filtrate was evaporated. The yellow oil was purified by silica gel column chromatography to give white solid compound 5a $(2.57 \mathrm{~g}, 85 \%)$.

m.p. $142-144{ }^{\circ} \mathrm{C} ;{ }^{1} \mathrm{H}$ NMR $\left(400 \mathrm{MHz}, \mathrm{CDCl}_{3}, 25^{\circ} \mathrm{C}\right)$ $\delta 7.93-6.69(\mathrm{~m}, 25 \mathrm{H}), 3.92(\mathrm{~d}, J=14.2 \mathrm{~Hz}, 6 \mathrm{H}), 3.80-$ $3.62(\mathrm{~m}, 6 \mathrm{H}), 3.30(\mathrm{~d}, J=2.0 \mathrm{~Hz}, 2 \mathrm{H}), 2.74(\mathrm{~s}, 4 \mathrm{H}) ;{ }^{13} \mathrm{C}$ NMR (100 MHz, DMSO, $\left.25^{\circ} \mathrm{C}\right) \delta 140.00$ (s), 138.66 (s), 128.71 (s), 128.18 (s), 128.07 (s), 127.91 (s), 126.93 (s), 126.55 (s), 96.10 (s), 74.23 (s), 59.83 (s), 56.41 (s), 51.74 (s); IR $\left(\mathrm{KBr}, v / \mathrm{cm}^{-1}\right)$ 3061, 3024, 2900, 2800, 1602, 1493, 1453, 773, 698; HRMS $\left(\mathrm{ESI}^{+}\right)$: calcd. for $\mathrm{C}_{41} \mathrm{H}_{43} \mathrm{~N}_{5}$ $[\mathrm{M}+\mathrm{H}]^{+} 606.3591$, found 606.3589 .

\subsection{2,4,6,8-(4-Methyl)benzyl-10-benzyl- $2,4,6,8,10$-pentaaza [3.3.3]propellane (5b)}

4b was replace of $\mathbf{4 a}$, the synthesis of compound $\mathbf{5 b}$ (78\%) was the same as the compound $\mathbf{5 a}$.

m.p. 122.7 - $125.5{ }^{\circ} \mathrm{C}$; ${ }^{1} \mathrm{H}$ NMR $\left(400 \mathrm{MHz}, \mathrm{CDCl}_{3}\right.$, $\left.25^{\circ} \mathrm{C}\right) \delta 7.52-6.77(\mathrm{~m}, 21 \mathrm{H}), 3.81(\mathrm{~d}, J=19.9,8.6 \mathrm{~Hz}$, $6 \mathrm{H}), 3.60(\mathrm{~d}, J=13.2 \mathrm{~Hz}, 6 \mathrm{H}), 3.19(\mathrm{~d}, J=3.5 \mathrm{~Hz}, 2 \mathrm{H})$, $2.63(\mathrm{~s}, 4 \mathrm{H}), 2.17(\mathrm{~d}, J=23.8 \mathrm{~Hz}, 12 \mathrm{H}) ;{ }^{13} \mathrm{C}$ NMR $(100$ $\left.\mathrm{MHz}, \mathrm{CDCl}_{3}, 25{ }^{\circ} \mathrm{C}\right) \delta 135.87$ (s), 135.12 (s), 127.85 (s), 127.26 (s), 126.93 (s), 126.00 (s), 95.09 (s), 73.46 (s), $58.94(\mathrm{~s}), 55.52$ (s), 50.51 (s), $20.04(\mathrm{~s})$; IR $\left(\mathrm{KBr}, \mathrm{v} / \mathrm{cm}^{-}\right.$ 1) $3022.45,2916.37,2893.22,2819.93,2779.42,2696.48$, $1514.12,1452.40,1377.17,1359.82,1290.38,1261.45$, $1174.65,1101.35,1020.34,877.61,798.53,742.59$,
698.23, 476.42; HRMS $\left(\mathrm{ESI}^{+}\right)$calcd. for $\mathrm{C}_{45} \mathrm{H}_{51} \mathrm{~N}_{5}$ $[\mathrm{M}+\mathrm{H}]^{+} 662.4217$, found 662.4220 .

\section{$3.9 \quad 2,4,6,8-(4-F l u o r o)$ benzyl-10-benzyl- 2,4,6,8,10-pentaaza [3.3.3] propellane (5c)}

$4 \mathbf{c}$ was replace of $\mathbf{4 a}$, the synthesis of compound $\mathbf{5 c}(72 \%)$ was the same as the compound $\mathbf{5 a}$.

m.p. $111.2-113.0{ }^{\circ} \mathrm{C} ;{ }^{1} \mathrm{H}$ NMR $\left(400 \mathrm{MHz}, \mathrm{CDCl}_{3}\right.$, $\left.25{ }^{\circ} \mathrm{C}\right) \delta 7.46-6.66(\mathrm{~m}, 22 \mathrm{H}), 3.75(\mathrm{~d}, J=13.8 \mathrm{~Hz}, 6 \mathrm{H})$, $3.68-3.49(\mathrm{~m}, 6 \mathrm{H}), 3.15(\mathrm{~d}, J=4.5 \mathrm{~Hz}, 2 \mathrm{H}), 2.61$ (s, $4 \mathrm{H}) ;{ }^{13} \mathrm{C} \mathrm{NMR}\left(100 \mathrm{MHz}, \mathrm{CDCl}_{3}, 25{ }^{\circ} \mathrm{C}\right) \delta 161.99$ (s), 159.56 (s), 137.47 (s), 134.40 (d), 128.33 (d), 127.86 (s), 127.34 (s), 127.34 - 126.66 (m), 126.18 (s), 95.04 (s), 58.90 (s), $55.26(\mathrm{~s}), 50.04(\mathrm{~s})$; IR $\left(\mathrm{KBr}, \mathrm{v} / \mathrm{cm}^{-1}\right) 3035.96$, 2912.51, 2806.43, 1602.85, 1508.33, 1452.40, 1375.25, $1220.94,1151.50,1091.71,1014.56,848.68,821.68$, 700.16; HRMS (ESI ${ }^{+}$): calcd. for $\mathrm{C}_{41} \mathrm{H}_{39} \mathrm{~F}_{4} \mathrm{~N}_{5}[\mathrm{M}+\mathrm{H}]^{+}$ 678.3214 , found 678.3225 .

\section{Conclusion}

In summary, the process route of 2,4,6,8,10-pentabenzyl2,4,6,8,10-pentaaza[3.3.3]propellane 5a was improved, and 2,4,6,8,10-pentaaza[3.3.3]propellane substituted with different groups, such as $\mathbf{5 b}$ and $\mathbf{5 c}$, were synthesized based on the condensation of 3,7,9,11-tetraoxo-2,4,6,8(4-methyl)benzyl-10-benzyl-2,4,6,8,10-pentaaza[3.3.3] propellane $\mathbf{4 b}$ and 3,7,9,11-tetraoxo-2,4,6,8-(4fluoro)benzyl-10-benzyl-2,4,6,8,10-pentaaza-

[3.3.3]propellane 4c. The further studies of 2,4,6,8,10pentaaza[3.3.3] propellanes substituted with different groups are in progress now.

\section{Acknowledgments}

This work was supported by the National Natural Science Foundation of China (No. 41471198) and the National Key Basic Research Program of China (No. 2014CB138801).

\section{References}

1. Weiss, U., Edwards, J.M. (1968) A one-step synthesis of ketonic compounds of the pentalane, $[3,3,3]-$ and $[4,3,3]$-propellane series. Tetrahedron. Lett., 9: 4885-4887.

2. Altman, J., Babad, E., Itzchaki, J., Ginsburg, D. (1966) Propellanes-I. Tetrahedron., 22: 279-304.

3. Wiberg., Kenneth, B. (1983) Strain energies of small ring propellanes. J. Am. Chem. Soc., 105: 12271233.

4. Weber, R.W., Cook, J.M. (1978) General method for the synthesis of [ n. 3.3]propellanes, $n \geqslant 3$. Can. J. Chem., 56: 189-192.

5. Ioffe, A.I., Svyatkin, V.A., Nefedov, O.M. (1988) Molecular-mechanical analysis of the structure of strained organic molecules. 6. [m.n.k]-Propellanes. Russ. Chem. Bull., 37: 1827-1836. 
6. Nguyen, T.X., Kobayashi, Y. (2008) Synthesis of the common propellane core structure of the hasubanan alkaloids. J. Org. Chem., 73: 5536-5541.

7. Yamamoto, N., Fujii, H., Nemoto, T., Nakajima, R., Momen, S., Izumimoto, N., Hasebe, K., Mochizuki, H., Nagase, H. (2011) Synthesis of new opioid derivatives with a propellane skeleton and their pharmacology: part 1. Bioorg. Med. Chem. Lett., 21: 4104-4107.

8. Navarro, R., Reisman, S.E. (2012) Rapid construction of the aza-propellane core of acutumine via a photochemical $[2+2]$ cycloaddition reaction. Org. Lett., 14: 4354-4357.

9. Eichberg, M.J., Dorta, R.L., Grotjahn, D.B., Lamottke, K., Schmidt, M., Vollhardt, K.P. (2001) Approaches to the synthesis of (+/-)-strychnine via the cobalt-mediated $[2+2+2]$ cycloaddition: rapid assembly of a classic framework. J. Am. Chem. Soc., 123 9324-9337.

10. Ibuka, T., Tanaka, K., Inubushi, Y. (1974) Total Synthesis of the Alkaloid, $( \pm)$-Hasubanonine. Chem. Pharm. Bull., 22: 782-798.

11. Zu, L., Boal, B.W., Garg, N. K. (2011) Total Synthesis of $( \pm)$-Aspidophylline A. J. Am. Chem. Soc., 133: 8877-8879.

12. Jian, M.H., Ritsuko, Y., Chun, S.Y.(2000) Solid phase synthesis of 1,3-disubsituted succinimides. Tetrahedron Lett., 41: 6111-6114.

13. Dilmaç, A.M., Wezeman, T., Bär, R.M., Bräse, S. (2020) Occurrence, synthesis and applications of natural and designed [3.3.3]propellanes. Nat. Prod. Rep. 37: 224-245.

14. Ginsburg, D. (1975) Propellanes-structure and reactions. VCH Publishing, Weinheim.

15. Zalkov, L.H., Harris, R.N.I., Derveer, D.V. (1978) Modhephene: a sesquiterpenoid carbocyclic [3.3.3]propellane. X-Ray crystal structure of the corresponding diol. J. Chem. Soc. Chem. Commun., 10: $420-421$

16. Smith, A.B.III., Jerris, P.J. (1981) Total synthesis of (+/-)-modhephene. J. Am. Chem. Soc., 103: 194195.

17. Lee, H.Y., Kim, S., Kim, D.I. (1996) Tandem radical cyclization reation of $\mathrm{N}$-aziridinyl imines to $[3,3,3]$ propellanes: formal total synthesis of dimodhephene. J. Am. Chem. Soc., 61: 1539-1540.

18. Burnett, C.A., Lagona, J., Wu, A.X., Shaw, A.J., Coady, D., Fettinger, J.C., Day, A.I., Isaacs, L. (2003) Preparation of glycoluril monomers for expanded cucurbit[n] uril synthesis. Tetrahedron., 59: 1961-1970.

19. Knowles, P., Harris, N.V. (1983) A. synthesis of 3,7,10-triazatricyclo[3.3.3.01,5] undecane, 3,7,10triaza-[3.3.3]propellane, and some derivatives. J. Chem. Soc. Perkin. Trans. I., 7: 1475-1477.

20. Olga, W., Philip, K., David, G. (1985) Propellanes. Part LXXVIII. Preparation of Several Triaza[3.3.3]propellanes and an
Oxadiaza[3.3.3]propellane. Helv. Chim. Acta., 68: 610-613.

21. Zhang, Q.H., Zhang, J.H., Qi, X.J., Shreeve, J.M. (2014) Molecular design and property prediction of high density polynitro [3,3,3]-propel-lanederivatized frameworks as potential high explosives. J. Phys. Chem. A., 118: 10857-10865.

22. Shin, M., Kim, M.H., Ha, T.H., Jeon, J., Chung, K.H., Kim, J.S., Kim, Y.G. (2014) Synthesis of novel 2,4,6,8,10-pentaaza[3.3.3]propellane derivativs. Tetrahedron., 70: 1617-1620. 